\title{
Single-cell RNA Sequencing Reveals That Injected ADSCs Change the Macrophage Profile in a Murine Model of Pulmonary Fibrosis
}

\section{Mamatali Rahman}

Tsinghua University School of Life Sciences

\section{Zhao-Yan Wang}

Tsinghua University School of Life Sciences

Jun-Xiang Li

Tsinghua University School of Life Sciences

Hao-Wei Xu

Tsinghua University School of Life Sciences

Qiong Wu ( $\nabla$ wuqiong@mail.tsinghua.edu.cn )

Tsinghua University School of Life Sciences https://orcid.org/0000-0002-9048-9094

\section{Research Article}

Keywords: adipose-derived mesenchymal stem cells (ADSCs), idiopathic pulmonary fibrosis (IPF), bleomycin (BLM), macrophage, scRNA-seq

Posted Date: August 2nd, 2021

DOl: https://doi.org/10.21203/rs.3.rs-744955/v1

License: (c) (i) This work is licensed under a Creative Commons Attribution 4.0 International License. Read Full License 


\section{Abstract}

Background: Idiopathic pulmonary fibrosis (IPF) is a deadly chronic interstitial lung disease with no effective treatment options other than lung transplantation. Allogeneic adipose-derived mesenchymal stem cells (ADSCs) are considered ideal as seed cells for stem cell-based therapy, and some studies illustrated the therapeutic effect of ADSCs on IPF, but the underlying mechanisms remain unclear.

Methods: A single intratracheal dose of bleomycin (BLM) was administered to induce pulmonary injury/fibrosis in C57BL/ 6 mice, after GFP-labeled mouse ADSCs (mADSCs) were implanted intratracheally to explore their potential therapeutic effects in the inflamed/fibrotic lung microenvironment. The mADSCs were then retrieved through fluorescence-activated cell sorting and subjected to single-cell RNA sequencing (scRNA-seq).

Results: Our data indicate that the single-dose intratracheal administration of mADSCs could significantly increase the life span of IPF mice by remodeling the extracellular matrix and promoting the polarization of macrophages to an anti-inflammatory phenotype.

Conclusions: A single intratracheal injection of mADSCs alleviated BLM-induced pulmonary fibrosis by readjustment of the mouse lung microenvironment, which was reflected in changes of the lung $\mathrm{C}_{\mathrm{Q}} \mathrm{QB}^{+}$, $\mathrm{APOE}^{+}$and TREM2 ${ }^{+}$macrophages in the mouse model.

\section{Background}

Idiopathic pulmonary fibrosis (IPF) denotes a heterogeneous group of severe and lethal interstitial lung diseases, involving progressive lung remodeling, alveolar destruction, excessive pathological extracellular matrix (ECM) deposition, and scarring of the lungs [1-3]. This devastating condition results from a dysregulated wound healing process, which culminates in the disruption of basic gas exchange in the lungs causing pulmonary insufficiency and ultimately leading to respiratory failure [4]. The average survival time of IPF patients is around 2-3 years after diagnosis [5]. The etiology and pathogenic mechanisms underlying IPF are complex and have not been completely elucidated, but the pathogenesis of IPF can be classified into the three stages of predisposition, initiation, and disease progression [6, 7]. Various factors predisposing to pulmonary fibrosis comprise genetic factors, environmental exposure to certain agents, chemotherapy, aging, and other influences $[6,8]$. Moreover, the pathogenesis involves multiple pathways, including the inflammatory response, apoptosis, oxidative stress, and epithelial to mesenchymal transition [9-13].

IPF is traditionally considered to be an irreversible lung disease that is refractory to various treatments, resulting in high rates of mortality and morbidity [14]. Recently, mesenchymal stem cells (MSCs) have emerged as a promising regenerative therapy for fibrotic diseases such as pulmonary fibrosis [15]. Adult MSCs have been isolated from various adult tissues such as the bone marrow, adipose tissue, and the stroma of organs. They exhibit self-renewal and the ability to differentiate into a variety of cell lineages, including chondrocytes, osteoblasts, and adipocytes $[16,17]$. Adipose-derived mesenchymal stem cells 
(ADSCs) have advantages of easy accessibility, relative abundance, and rapid proliferation ability compared to bone marrow MSCs, which makes them ideal seed cells for autologous cell therapy $[18,19]$. ADSCs have antifibrotic, antiapoptotic, antioxidant, and immunoregulatory effects in the microenvironment affected by different fibrotic diseases, in which they attenuate the expression of fibrogenic cytokines and collagen types. ADSCs were found to stimulate the polarization of macrophages into the M2 phenotype by secreting IL- 6 in the inflammatory environment of peritoneal fibrosis [20], or by activating the PI3K/STAT3-dependent pathway in cardiac fibrosis [21]. They were also reported to suppress the infiltration of macrophages and T cells into the injured side in a bleomycin-induced scleroderma model [22], prevent leucocyte infiltration and modulate the expression of IL-1b, TNF-a, IL-6 in a combined model of peritoneal fibrosis and chronic kidney disease [23]. Additionally, ADSCs increased the number of type II alveolar epithelial cells in a radiation-induced lung fibrosis model [24], and ameliorated radiation-induced fibrosis by secreting HGF to downregulating the expression of TGF $\beta$, CTGF, IL-1, TNF and NF-KB, as well as recruiting BMSCs to the injured site [25]. Similarly, airway transplantation of ADSCs was found to protect mice against BLM-induced pulmonary fibrosis [26]. In vitro studies indicated that conditioned medium from ADSCs inhibits the activity of keloid fibroblasts and fibrosis by paracrine effects [27], while ADSCs were found to decrease apoptosis and fibrosis by secreting IGF1 in a cardiomyocyte damage model [28].

Although the effects of ADSCs have been widely investigated using both in vitro cultures and in vivo models, direct recovery and analysis of injected ADSCs were not performed yet. To successfully repair pulmonary fibrosis using adult stem cells like ADSCs, it is necessary to identify the therapeutic mechanisms of injected ADSCs. In this study, we directly retrieved injected mouse ADSCs (mADSCs) from the lungs of BLM-treated mice and subjected them to scRNA-seq to analyze the crosstalk between injected mADSCs and pulmonary resident cells and understand the mechanisms behind the anti-fibrotic effect of mADSCs.

\section{Materials And Methods}

\subsection{Animals and experimental design}

All the animal experiments were conducted under the authorization of the Institutional Animal Care and Use Committee of Tsinghua University. Six to eight-week-old male C57BL/6J mice, purchased from the Laboratory Animal Research Center of Tsinghua University, were housed in the specific pathogen-free experimental animal environment at the Laboratory Animal Research Center of Tsinghua University, $5 \sim 6$ mice per cage with sterilized feed and water ad libitum and a 12/12 hours light/dark cycle. The mice were randomly divided into three groups: (i) control group mock-treated with PBS, (ii) BLM + PBS group, and (iii) BLM + mADSCs group.

\subsection{Culture of adipose-derived mesenchymal stem cells}

GFP-labeled mouse adipose-derived mesenchymal stem cells (mADSCs) were obtained from Cyagen Biosciences (China) and employed in all the experiments, in vitro, and in vivo. The mADSCs were cultured 
in mouse adipose-derived mesenchymal stem cell basal medium (BM, SYAGEN, China) in a humidified atmosphere comprising $5 \%$ carbon dioxide at $37^{\circ} \mathrm{C}$. The culture medium was changed every two or three days. When the confluence reached $80 \sim 90 \%$, the mADSCs were detached using $0.25 \%$ trypsin (with $0.02 \%$ EDTA) and split at a ratio of 1:2. The cell culture experiments were performed using ADSCs at passage $3 \sim 6$.

\subsection{Construction of the BLM-induced pulmonary fibrosis mouse model and administration of mADSCs}

Mice were anesthetized with $2.5 \%$ tribromoethanol $(0.8 \% \mathrm{NaCl}, 1 \mathrm{mM}$ Tris $(\mathrm{pH} 7.4), 0.25 \mathrm{mM}$ EDTA (pH 7.4 ) ) and $3.5 \mathrm{mg} / \mathrm{kg}$ bleomycin (BLM) solution (Sigma B5507-15un) in 50 $\mu \mathrm{L}$ of PBS was administered intratracheally on day 0 using a $1 \mathrm{~mL} / \mathrm{cc}$ syringe with a $25 \mathrm{G}$ needle with insertions between the cartilaginous rings of the trachea. Control animals were treated with saline instead of BLM in the same manner as reported previously $[26,29]$. These procedures were performed in a sterile environment. The mADSCs implantation was performed on mice exposed to BLM for 72 hours. On day 3,50 $\mathrm{LL}$ of a suspension containing $1 \times 10^{7}$ cells $/ \mathrm{mL}$ in PBS $\left(5 \times 10^{5}\right.$ cells/mouse) was injected intratracheally using a $1 \mathrm{~mL}$ disposable syringe with a $25 \mathrm{G}$ needle. For the vehicle (PBS) group, $50 \mu \mathrm{L}$ PBS was injected instead. Before implantation, cells were washed three times to remove the culture medium. The duration of the study was set at four weeks, as the pulmonary fibrosis remodeling process in mice has been reported to be mostly complete (70 80\%) within 3 weeks. All animals were weighed daily from day 0 to 20 .

\subsection{Measurements of BLM-induced lung injury, histological evaluation of lung damage and collagen deposition}

To assess the development of pulmonary fibrosis, mice were assessed at 7 and 14 days after BLM, saline, and mADSCs administration. For the lung tissue collection, mice were euthanized by $\mathrm{CO}_{2}$ inhalation. The chest cavity was exposed and the lungs were perfused with ice-cold PBS via the ventricle until the tissue visibly whitened due to the washing out of blood. Then, the lungs were washed with icecold PBS to clean the remaining surface blood. Left lung tissue was fixed with $4 \%(\mathrm{v} / \mathrm{v})$ paraformaldehyde in PBS and embedded in paraffin before being cut into $5-\mu \mathrm{m}$-thick sections and mounted on glass slides. For histological observation and assessment of fibrosis accumulation in lung tissue, slices were stained with hematoxylin and eosin (H\&E) or Masson's trichrome stain, respectively. Then, we used an automatic digital slide-scanning system (Axio Scan.Z1, Zeiss, Germany) to scan the samples with the $20 \times$ objective. All the images were processed with ZEN (2.3) and lung tissue parenchyma density for grading the fibrosis level was calculated using IPP (Image-Pro premier 3D 9.2.0) software using random and non-overlapping fields.

\subsection{Preparation of single-cell suspensions}

For the preparation of single-cell suspensions of mouse lung tissue, mice were sacrificed on the 4th day following mADSCs treatment and the lungs were perfused with PBS. All samples, mADSCs- and PBStreated, were divided into single-cell suspensions by combining mechanical dissociation with enzymatic 
degradation of the extracellular matrix. The samples were enzymatically digested using the Lung Dissociation Kit, mouse (130-095-927, Miltenyi Biotec, Germany) according to the manufacturer's instructions. Briefly, tissues were cut into small pieces, which were mixed with $200 \mu \mathrm{L}$ of enzyme $\mathrm{H}, 25 \mu \mathrm{L}$ of enzyme A, $100 \mu \mathrm{L}$ of enzyme R, and $4.7 \mathrm{~mL}$ Dulbecco's Modified Essential Medium in a MACS C Tube (130-094392, Miltenyi Biotec). In the mechanical dissociation step, we used the gentle MACS ${ }^{\text {TM }}$

Dissociator (130-093-235, Miltenyi Biotec) three times and the samples were incubated for $30 \mathrm{~min}$ at $37^{\circ} \mathrm{C}$ at each dissociation step. Cells were passed through 70 and $40 \mu \mathrm{m}$ strainers to remove large pieces, after which the Red Blood Cell Lysis Solution (10x) (Sigma Aldrich, St. Louis, MO, USA) was used to eliminate erythrocytes, followed by FACS sorting of pulmonary resident cells and GFP-mADSCs, respectively.

\subsection{Preparation of the single-cell RNA sequencing (scRNA- seq) library}

We prepared the scRNA-seq libraries using the Chromium Next GEM Single Cell 3囚 Reagent kit (version 3.1) (10x Genomics, USA). Single-cell suspensions were loaded onto the Chromium Single Cell Controller Instrument (10x Genomics) to generate single-cell gel beads in emulsions. Then, reverse transcription was used to generate barcoded full-length cDNA followed by the disruption of emulsions using the recovery agent. To clean up the cDNA, Dynabeads Silane magnetic beads (Thermo Fisher Scientific) were used. Subsequently, the amplified cDNA was fragmented, end-repaired, A-tailed, index adapter-ligated, and library amplified. Libraries were sequenced on a HiSeq Xten VS NovaSeq 6000 sequencing platform (Illumina, USA), which generated $150 \mathrm{bp}$ paired-end reads.

\section{7 scRNA-seq data processing}

The scRNA-seq library sequences were de-multiplexed, mapped to the mouse genome, and counted to generate a gene expression matrix using CellRanger (10x Genomics). Further data processing was done using the R packages Seurat3 (UMAP visualization, clustering, and gene expression plots), ReactomePA (gene ontology enrichment analysis), and CellChat (cell-cell ligand-receptor interaction analysis).

\subsection{Immunofluorescence staining}

For immunofluorescence staining of SPP1, APOE, and TREM2 in the (ii) BLM + PBS and (iii) BLM + mADSCs groups, mice were anesthetized and lungs were extracted on days 7 and 10 . The left lung lobes were fixed with $4 \%(v / v)$ paraformaldehyde, paraffin-embedded and cut into 5 - $\mu \mathrm{m}$-thick sections.

\subsection{Real-time quantitative PCR}

To assess the gene expression levels, mice were euthanized for lung tissue collection and the total RNA was extracted using TRIZOL reagent (Invitrogen, USA) and reverse-transcribed into CDNA using the SuperScript II First-Strand Synthesis System Kit (GenStar, A215, china). The RT-PCR reactions were conducted using $2 \times$ RealStar Green Fast Mixture (GenStar, A304, china). GAPDH served as the internal reference gene and data were analyzed using the $2^{-} \triangle \triangle \mathrm{CT}$ method. The specific primers were as follows: TNF- $a$ (sense, 5'-CATCTTCTCAAAATTCGAGTGACAA-3'; anti-sense, 5'-TGGGAGTAGACAAGGTACAACCC3'), L-1 (sense, 5'-AGGTCGCTCAGGGTCACAAG-3'; anti-sense, 5'-GTGCTGCCTAATGTCCCCTTGAATC-3'), 
IL-10 (sense, 5'-TAAGGCTGGCCACACTTGAG-3'; anti-sense, 5'-GTTTTCAGGGATGAAGCGGC-3'), IL-6 (sense, 5'-GGTACATCCTCGACGGCATCT-3'; anti-sense, 5'-GTGCCTCTTTGCTGCTTTCAC-3'), and GAPDH (sense, 5'-GTAGTTGAGGTCAATGAAGGG-3'; anti-sense, 5'-TCGTCTCATAGACAAGATGGT-3'). The experiment was repeated three times in triplicate.

\subsection{Statistical analysis}

All the experiments were performed in at least three independent replicates, and data are presented as means $\pm S D$ and visualized using OriginPro or $R$ software. The statistical significance of differences was assessed using two-way ANOVA followed by Tukey's multiple comparisons tests; ${ }^{\star} P<0.05 ; * \star P<0.01$.

\section{Results}

\subsection{Intratracheal injection of mADSCs delayed the progression of pulmonary fibrosis}

In this study, we used the BLM-induced pulmonary fibrosis mouse model. We delivered $3.5 \mathrm{mg} / \mathrm{kg} \mathrm{BLM}$ to the lungs through the trachea of $5 \sim 6$-week-old male C57BL/6 mice with $50 \mu \mathrm{L}$ phosphate-buffered brine (PBS), based on a method reported previously [30]. To evaluate the effect of intratracheal/tail vein injection of mouse adipose-derived mesenchymal stem cells (mADSCs) on lung fibrosis, we injected $5 \times 10^{5}$ GFP-labeled mADSCs into the mouse lung with $50 \mu \mathrm{L}$ PBS at day 3 post BLM injection. The control group was injected with the same volume of PBS. We examined the survival of the mice during 45 days after BLM treatment. A single intratracheal injection of mADSCs significantly prolonged the survival of the mice compared to the control (Fig. 1b). We quantitatively assessed the fibrosis and alveolar damage in the lungs by histological staining, which revealed a visible improvement in the lung texture in the mADSCs group on day 14, but not on day 7 (Fig. 1c, d). The H\&E and Masson's trichrome stained slides were scanned, followed by automated quantification of the percentage of lung parenchyma and fibrotic area (Fig. 1e, f). A single injection of mADSCs significantly delayed the fibrosis of mouse lung tissues, the fibrotic area of the BLM + mADSCs group was $0.4 \%$ on day 14 , compared to $1.1 \%$ in the BLM + PBS group.

\subsection{Single-cell transcriptomics of mADSC-treated and untreated pulmonary resident cells}

In order to understand the effects of mADSCs in the inflammatory lung microenvironment, we compared the single-cell transcriptomic profiles of pulmonary resident cells from the BLM + mADSCs group with those from the BLM + PBS group. We injected the GFP-labeled mADSCs intratracheally into BLM-treated mice on day 3 , and the mADSCs and lung parenchyma cells were sorted from mouse lungs on day 7 by flow cytometry (Fig. 2a).

Firstly, we compared single-cell profiles of mouse lung parenchyma cells from the BLM + PBS and BLM + mADSCs groups (Fig. 2b), with libraries comprising 8181 and 6405 cells, respectively. A total of 27 cell clusters were identified in each group of lung parenchyma cells (supplementary Fig. 1a, b), and we 
identified 12 discrete cell types for each group based on cell markers with no cell cycle effects on data architecture (Fig. 2b). The identified lung cell types included pneumocytes, bronchiolar exocrine cells, natural killer cells, fibroblasts/epithelial cells, myofibroblasts, dendritic cells, endothelial cells, T cells, B cells, monocytes/macrophages, and granulocytes (Fig. 2b, c). Notably, the immune cells mainly included monocytes/macrophages in the BLM + mADSCs group (48.78\%), and granulocytes in the LM + PBS group (44.87\%) (Fig. 2d). Fibroblasts, endothelial cells and myofibroblasts were the main non-immune cells in the BLM + mADSCs group, accounting for $7.45,6.83$, and $3.80 \%$, respectively.

Monocytes/macrophages were the dominant cell type in both the BLM + mADSCs group (48.78\%) and the BLM + PBS group (27.28\%) (Fig. 3a), and were clustered into 9 subclasses based on their gene expression profiles (Fig. 3b). The Mo/Ma-1 subclass, characterized by the expression of the marker genes MPG, COL1a1, and SPARC, was the most abundant subpopulation (48.71\%). The macrophage-4 subclass, expressing GPNMB, PF4, and APOE, was the second most abundant subpopulation in the BLM + mADSCs group $(20.42 \%)$. By contrast, the alveolar macrophage-21 and the Mo/Ma-24 subclasses were practically not detectable in the BLM + PBS group (Fig. 3b), 0 and $0.07 \%$, respectively.

We next examined the function of genes exhibiting significant changes of expression levels in the Mo/Ma-1 and macrophage-4 subclasses based on gene ontology (GO) and KEGG pathways analysis (Fig. 3c, d). The results indicated that the Mo/Ma-1 subclass was related to the regulation of the extracellular matrix (ECM) and proteoglycans, non-integrin membrane-ECM interactions, collagen chain trimerization, collagen degradation, and crosslinking of collagen fibrils, with high expression of COL1a1, COL1a2, and SPARC genes.

\section{3 scRNA-seq of mADSCs retrieved from mouse lungs}

To investigate the potential therapeutic mechanism of intratracheally injected mADSCs in the BLMinjured inflammatory lung microenvironment, we analyzed the single-cell transcriptomic profiles of retrieved mADSCs from mouse lungs on day 7, i.e., 4 days post-injection of mADSCs. We intratracheally injected the GFP-labeled mADSCs ( $5 \times 10^{5}$ cells in $50 \mu \mathrm{L}$ of PBS per mouse) into BLM-treated mice on day 3 and the mADSCs were retrieved from mouse lungs on day 7 by fluorescence-activated cell sorting (FACS). On day 7 , the moue lung was extracted and a single-cell suspension was prepared by enzymatic digestion to retrieve the GFP-expressing mADSCs by FACS. The retrieved mADSCs were subjected to for $10 \times$ scRNAseq analysis, with in-vitro cultured mADSCs as control.

We retrieved a total of $417 \mathrm{GFP}+$ cells from three mice lungs and pooled them together. At the same time, 2801 culture cells were sorted, and a single mRNA sequencing library was constructed for each group. When comparing the retrieved mADSCs with in-vitro cultured cells using UMAP cluster analysis, we found a great heterogeneity among the retrieved mADSCs (Suppl. Figure 2a). The gene expression pattern of retrieved and in-vitro cultured mADSCs is highlighted in Suppl. Figure 2d. Notably, the retrieved mADSCs exhibited high expression level of COL6a1/2/3, TNC, AAP1, FOSB, CRIP1, and COL3a1, which were practically not expressed by in-vitro cultured mADSCs. 
We further analyzed the functions of genes with significantly changed expression levels in the retrieved mADSCs using gene ontology (GO) analysis. The upregulated genes of the retrieved mADSCs were found to be related to ECM remodeling in the BLM-injured lung microenvironment (Suppl. Figure 2b), with GO terms such as Extracellular matrix organization, Degradation of the extracellular matrix, Collagen formation, Assembly of collagen fibrils and other multimeric structures, Collagen degradation, Collagen biosynthesis and modifying enzymes, Collagen chain trimerization, as well as Non-integrin membraneECM interactions pathways reflected in the significant upregulation of $A P P, B M P 1, C D 44, C O L 2 a 1$, and COL5a 1 (Fig. 2d).

\section{4 mADSCs alleviate lung fibrosis by promoting the polarization of macrophages to an anti-inflammatory phenotype and downregulating pro-inflammatory cytokines in the early stage}

Although the retrieved mADSCs did not show significant expression of anti-inflammatory cytokines, we were able to confirm that the abundance of TREM $2^{+}$macrophages (cluster 4 identified by scRNA-seq) was increased at day 7 in the BLM + mADSCs group, as indicated by the stronger fluorescence signals of TREM2 and SPP1(OPN) (Fig. 4a). However, APOE, which was highly expressed by most of the monocyte/macrophage subgroups, was not found to be upregulated, suggesting that it may not participate in the anti-inflammatory activity of these macrophages.

Similarly, qPCR showed that the pro-inflammatory cytokines tumor necrosis factor-alpha (TNF-a) and interleukin-1 beta (IL-1 $\beta$ ) were significantly downregulated in the mouse lungs at day 7, while antiinflammatory or functional cytokines IL-10 and IL-6 exhibited the opposite trend (Fig. 4b).

To investigate the interplay between the injected mADSCs and the lung cells in the BLM-injured lung microenvironment, we first analyzed the lung cells and retrieved mADSCs together, and visualized their outgoing and ingoing communication patterns. We identified the global communication patterns and the key signals in different cell subgroups by employing the pattern-recognition algorithm CellChat. The four outgoing communication patterns were analyzed and four incoming communication signals were identified (Suppl. Figure 3a). Pattern \#1 accounted for the largest portion of outgoing signaling, which represents TGF- $\beta$, GDF, VEGF, XCR, and IL2, produced by immune cells such as Mo/Ma-20, macrophage-4, NK cell-17 and T-cell-23. The outgoing signaling of recovered mADSCs was assigned to pattern \#3, which included the ncWNT, EGF, PDGF, PTN, and PERIOSTIN signaling pathways (Suppl. Figure 3a). On the other hand, the incoming communication pattern of target cells showed that the injected mADSCs received BMP, WNT, EGF, FGF, PDGF, and OSM signals in the BLM-injured lung microenvironment, which are assigned to incoming communication pattern \#2 (Suppl. Figure 3b).

We detected 51 significant ligand-receptor pairs among the 28 cell groups/clusters including the retrieved mADSCs, which were categorized into NRG, PERIOSTIN, TGF- $\beta$, HGF, PDGF, TNF, WNT, non-canonical WNT 
(ncWNT), SPP1, PTN, CXCL, and CCL pathways. We next found that the retrieved mADSCs received Mo/Ma-20 subgroup signals via the NRG signaling network (Suppl. Figure 3c) Using network centrality analysis (NCA), and ITGAV/ITGB3 were identified as the contribution receptors on mADSCs in the BLMinjured lung microenvironment (Suppl. Figure 3d). Similarly, the PERIOSTIN signaling pathway was identified as the main communication channel from mADSCs to the Macrophage-4 subgroup (Suppl. Figure 3d). Postn-ITGAV/ITGB5 was the dominant contributing ligand-receptor pair (Suppl. Figure 3e). In addition to these pathways, the mADSCs were also sending HGF to plasmacytoid dendritic cell-11 and pneumocyte- 26 subgroups through the HGF signaling pathway, and HGF-MET was the main contributing ligand-receptor pair (data not shown).

\section{Discussion}

Lung fibrosis is one of the leading causes of mortality among patients with lung disease [31]. Moreover, nintedanib and pirfenidone remain the only drugs recommended for the treatment of pulmonary fibrosis in the current evidence-based guidelines [32,33], and they offer only limited efficiency in preventing the progression of fibrosis $[34,35]$. Previous publications have reported the beneficial effects of adiposederived mesenchymal stem cells (ADSCs) or their extracellular vesicles in both clinical trials and experimental animal models of fibrosis [36], such as those induced with BLM [26, 37-39], radiation [24], or PM2.5 [40], as well as lung silicosis [41], covid-19 related pulmonary fibrosis and fibrosis of other organs/tissues [21, 25, 42-48]. However, all these studies only investigated the therapeutic mechanism of ADSCs at the tissue level in vivo or in vitro, and to our knowledge, none studied the effects of ADSCs in the inflamed, fibrotic microenvironment at the single-cell level.

In this study, we intratracheally injected mouse ADSCs (mADSCs) into the lungs of bleomycin (BLM)injured male C57BL/6J mice and comprehensively characterized the transcriptions of retrieved mADSCs from the injured lungs at the single-cell level, as well as the cell type composition and intercellular communications between pulmonary resident cells and mADSCs. The GFP-expressing mADSCs that were retrieved from the lungs by FACS were compared with in-vitro cultured mADSCs. The injected mADSCs decreased collagen deposition and protected the alveolar architecture, thereby prolonging the survival of the mice. These results were similar to previous studies using animal models of pulmonary fibrosis [26, 37-39].

Pulmonary fibrosis induced by administering BLM to mimic human pulmonary fibrosis in rodents is characterized by the progressive accumulation of ECM, disruption of the alveolar architecture, epithelialmesenchymal transition, injury/apoptosis of alveolar cells, activation and proliferation of myofibroblasts and fibroblasts, as well as the activation of inflammation/apoptosis/fibrosis-related genes and signaling pathways [30]. Pulmonary injury and inflammation occur in the early stage of BLM treatment, and collagen accumulation follows 1 to 2 weeks after BLM challenges (Fig. 1c, d). A single intratracheal injection of BLM will generate pulmonary fibrosis and it can lead to significant mortality of mice in one month (Fig. 1b). Previous studies found that intratracheal, intravenous or intraperitoneal administration of ADSCs can ameliorate pulmonary fibrosis in BLM-induced animal models $[26,39,49,50]$. The injected 
dose differs among studies, based on the experimental animal or injection method. For example, Llontop et al. injected $2 \times 10^{6} \mathrm{ADSC}$ in $100 \mu \mathrm{L}$ of saline intratracheally to treat BLM-induced pulmonary SD rat model [26], while Tashiro et al. injected $5 \times 10^{5}$ ADSCs in $200 \mu \mathrm{L}$ of PBS through the tail vein in a BLMinduced mouse model of pulmonary fibrosis [39]. The injected ADSCs were enriched in the pulmonary capillaries or injured sites, and their therapeutic mechanisms involve changing the ECM deposition and regulating inflammation.

Both adaptive and innate immune mechanisms play an important role in the progression of fibrosis, including various types of immune cells such as T cells, neutrophils, and macrophages [51]. The singlecell profiles of mouse pulmonary resident cells sequenced in this study indicated that monocytes/macrophage were the most dominant cell type in the BLM + PBS and BLM + mADSCs groups, accounting for 27.28 and $48.78 \%$, respectively. Among the monocyte/macrophage subgroups, clusters 1 and 4 were predominant in the BLM + mADSCs group, while immunofluorescence only indicated that the abundance of cluster 4 macrophages increased after mADSCs treatment of BLM-induced lung fibrosis.

The macrophage-4 cluster is characterized by high expression levels of SPP1 and TREM2. The SPP1 signaling pathway was also commonly found in the crosstalk among cell groups in the BLM + mADSCs scRNA-seq results, suggesting its potential role in mADSCs-treated lung tissue. SPP1 protein is crucial for mouse lung development [52], and a correlation between its expression and the severity of human IPF has also been observed. It was found that SPP1 may play a pro-fibrotic role by downregulating MMP-1 while upregulating MMP-7 [53]. However, the increased expression of SPP1 in this study occurred in the $B L M+m A D S C s$ group, which had better survival than the non-treated BLM + PBS group. This could mean that SPP1 is not the main determinant of BLM-induced lung fibrosis in mice, and its pro-fibrotic influence might have been counteracted by other MSC-based mechanisms. Similarly, TREM2 expression is also positively linked to the severity of lung diseases such as viral infection [54] and chronic obstructive pulmonary disease (COPD) [55], and it is believed to suppress pro-inflammatory responses [54,56]. The macrophage-4 subgroup, which increased in abundance after mADSCs administration, might therefore have alleviated the early inflammation caused by BLM and postponed, though not prevented, the subsequent fibrotic response.

\section{Conclusions}

In conclusion, this study demonstrates that intratracheal injection of mADSCs can delay bleomycin (BLM)-induced lung fibrosis and improve lung function in mice. The mADSCs decreased collagen deposition and protected the alveolar architecture following BLM exposure. Furthermore, single-cell mRNA sequencing indicated that the injected mADSCs received NRG signals from Mo/Ma-20 monocytes and influenced the majority of monocyte/macrophage cells via the PTN pathway. The SPP1 ${ }^{+} /$TREM2 $^{+}$ anti-inflammatory macrophage-4 subgroup was significantly more abundant after mADSCs injection in BLM-treated lungs, which might be the key mechanisms through which mADSCs delayed/alleviated pulmonary fibrosis. Together with the overall findings of suppressed inflammation at day 7 , these results indicated that the injection of mADSCs reduced inflammation in the mouse lungs at the early stage of 
BLM-induced fibrosis. Our work did not provide how cell-cell communication among/between the injected mADSCs and the pulmonary resident cells to regulate pulmonary fibrosis in the murine model, maybe because this is not the right time to retrieve the mADSCs. But this study represents the first step towards the direct detection of seed cells mechanism in cell therapy.

\section{Abbreviations}

IPF: Idiopathic pulmonary fibrosis; ADSCs: Adipose-derived mesenchymal stem cells; BLM: Bleomycin; scRNA-seq; Single-cell RNA sequencing; ECM: Extracellular matrix; PBS: Phosphate buffered saline; IGF: Insulin-like growth factor; IGFBPs: Insulin-like growth factor binding proteins.

\section{Declarations}

\section{Acknowledgments}

M. Rahman and Z. Y. Wang contributed equally to this work. We thank the Laboratory Animal Research Center and Experimental Apparatus Sharing Platform of Tsinghua University (PR China) for providing technical support as well as experimental platforms.

\section{Funding}

This work was supported by Ministry of Science and Technology of China (Grant No. 2018YFA0900100), and National Natural Science Foundation of China (Grant No. 31961133019, 31670991, and 31470933).

\section{Authors' contributions}

Conceptualization, Q.W.; Methodology, M. Rahman, Z. Y. Wang, J.X Li and H.W. Xu; Investigation, M. Rahman, Z. Y. Wang and H.W. Xu; Software, M. Rahman and Z. Y. Wang; Formal analysis, M. Rahman and Z. Y. Wang; Writing-original draft, M. Rahman and Z. Y. Wang; Writing-review \& editing, Q.W.; Funding acquisition, Q.W.; Resources, Q.W.; Supervision, Q.W.. The authors read and approved the final manuscript.

\section{Availability of data and materials}

The data that support the findings of this study are available from the corresponding author upon reasonable request.

\section{Ethics approval}


All the animal experiments were conducted under the authorization of the Institutional Animal Care and Use Committee of Tsinghua University (Approval No. 18-WQ2).

\section{Consent for publication}

All authors of this manuscript agree to its publication.

\section{Competing interests}

The authors declare no competing interests.

\section{References}

1. Wuyts, W.A., et al., The pathogenesis of pulmonary fibrosis: a moving target. Eur Respir J, 2013. 41(5): p. 1207-18.

2. Habiel, D.M. and C. Hogaboam, Heterogeneity in fibroblast proliferation and survival in idiopathic pulmonary fibrosis. Front Pharmacol, 2014. 5: p. 2.

3. Shah, A. and C.D. Fell, Idiopathic Pulmonary Fibrosis, in Interstitial Lung Disease. 2018. p. 121-129.

4. Betensley, A., R. Sharif, and D. Karamichos, A Systematic Review of the Role of Dysfunctional Wound Healing in the Pathogenesis and Treatment of Idiopathic Pulmonary Fibrosis. J Clin Med, 2016. 6(1).

5. Ley, B., H.R. Collard, and T.E. King, Jr., Clinical Course and Prediction of Survival in Idiopathic Pulmonary Fibrosis. American Journal of Respiratory and Critical Care Medicine, 2011. 183(4): p. 431-440.

6. Wolters, P.J., H.R. Collard, and K.D. Jones, Pathogenesis of Idiopathic Pulmonary Fibrosis, in Annual Review of Pathology: Mechanisms of Disease, Vol 9, A.K. Abbas, S.J. Galli, and P.M. Howley, Editors. 2014. p. 157-179.

7. Sgalla, G., et al., Idiopathic pulmonary fibrosis: pathogenesis and management. Respir Res, 2018. 19(1): p. 32.

8. Abid, S.H., V. Malhotra, and M.C. Perry, Radiation-induced and chemotherapy-induced pulmonary injury. Curr Opin Oncol, 2001. 13(4): p. 242-8.

9. Wilkes, D.S., et al., Oral immunotherapy with type $V$ collagen in idiopathic pulmonary fibrosis. Eur Respir J, 2015. 45(5): p. 1393-402.

10. Richeldi, L., H.R. Collard, and M.G. Jones, Idiopathic pulmonary fibrosis. Lancet, 2017. 389(10082): p. 1941-1952.

11. King, T.E., Jr., A. Pardo, and M. Selman, Idiopathic pulmonary fibrosis. Lancet, 2011. 378(9807): p. 1949-1961.

12. Kinnula, V.L., et al., Oxidative stress in pulmonary fibrosis: a possible role for redox modulatory therapy. Am J Respir Crit Care Med, 2005. 172(4): p. 417-22. 
13. Rydell-Tormanen, K., et al., Extracellular matrix alterations and acute inflammation; developing in parallel during early induction of pulmonary fibrosis. Lab Invest, 2012. 92(6): p. 917-25.

14. Glasser, S.W., et al., Mechanisms of Lung Fibrosis Resolution. Am J Pathol, 2016. 186(5): p. 1066-77.

15. Gazdhar, A., et al., The secretome of induced pluripotent stem cells reduces lung fibrosis in part by hepatocyte growth factor. Stem Cell Res Ther, 2014. 5(6): p. 123.

16. Park, J.S., et al., The promotion of chondrogenesis, osteogenesis, and adipogenesis of human mesenchymal stem cells by multiple growth factors incorporated into nanosphere-coated microspheres. Biomaterials, 2011. 32(1): p. 28-38.

17. Ackema, K.B. and J. Charité, Mesenchymal stem cells from different organs are characterized by distinct topographic Hox codes. Stem Cells Dev, 2008. 17(5): p. 979-91.

18. Ivanova-Todorova, E., et al., Adipose tissue-derived mesenchymal stem cells are more potent suppressors of dendritic cells differentiation compared to bone marrow-derived mesenchymal stem cells. Immunol Lett, 2009. 126(1-2): p. 37-42.

19. Noel, D., et al., Cell specific differences between human adipose-derived and mesenchymal-stromal cells despite similar differentiation potentials. Exp Cell Res, 2008. 314(7): p. 1575-84.

20. Yang, C.Y., et al., Adipose-derived mesenchymal stem cells attenuate dialysis-induced peritoneal fibrosis by modulating macrophage polarization via interleukin-6. Stem Cell Res Ther, 2021. 12(1): p. 193.

21. Lee, T.M., et al., Preconditioned adipose-derived stem cells ameliorate cardiac fibrosis by regulating macrophage polarization in infarcted rat hearts through the PI3K/STAT3 pathway. Lab Invest, 2019. 99(5): p. 634-647.

22. Okamura, A., et al., Adipose-derived stromal/stem cells successfully attenuate the fibrosis of scleroderma mouse models. Int J Rheum Dis, 2020. 23(2): p. 216-225.

23. Costalonga, E.C., et al., Adipose-Derived Mesenchymal Stem Cells Modulate Fibrosis and Inflammation in the Peritoneal Fibrosis Model Developed in Uremic Rats. Stem Cells Int, 2020. 2020: p. 3768718.

24. Zhang, Y., X. Jiang, and L. Ren, Optimization of the adipose-derived mesenchymal stem cell delivery time for radiation-induced lung fibrosis treatment in rats. Sci Rep, 2019. 9(1): p. 5589.

25. Ejaz, A., et al., Adipose-Derived Stem Cell Therapy Ameliorates Ionizing Irradiation Fibrosis via Hepatocyte Growth Factor-Mediated Transforming Growth Factor-beta Downregulation and Recruitment of Bone Marrow Cells. Stem Cells, 2019. 37(6): p. 791-802.

26. Llontop, P., et al., Airway transplantation of adipose stem cells protects against bleomycin-induced pulmonary fibrosis. J Investig Med, 2018. 66(4): p. 739-746.

27. Liu, J., et al., Human adipose tissue-derived stem cells inhibit the activity of keloid fibroblasts and fibrosis in a keloid model by paracrine signaling. Burns, 2018. 44(2): p. 370-385.

28. Chen, T.S., et al., Adipose-derived stem cells decrease cardiomyocyte damage induced by porphyromonas gingivalis endotoxin through suppressing hypertrophy, apoptosis, fibrosis, and 
MAPK markers. Environ Toxicol, 2018. 33(4): p. 508-513.

29. Tzouvelekis, A., et al., Stem cell therapy for idiopathic pulmonary fibrosis: a protocol proposal. J Transl Med, 2011. 9(1): p. 182.

30. Walters, D.M. and S.R. Kleeberger, Mouse models of bleomycin-induced pulmonary fibrosis. Curr Protoc Pharmacol, 2008. Chapter 5: p. Unit 546.

31. Geiger, S., D. Hirsch, and F.G. Hermann, Cell therapy for lung disease. Eur Respir Rev, 2017. 26(144).

32. Raghu, G., et al., An Official ATS/ERS/JRS/ALAT Clinical Practice Guideline: Treatment of Idiopathic Pulmonary Fibrosis An Update of the 2011 Clinical Practice Guideline. American Journal of Respiratory and Critical Care Medicine, 2015. 192(2): p. E3-E19.

33. Spagnolo, P., A. Tzouvelekis, and F. Bonella, The management of patients with idiopathic pulmonary fibrosis. Frontiers in Medicine, 2018. 5(JUL).

34. Galli, J.A., et al., Pirfenidone and nintedanib for pulmonary fibrosis in clinical practice: Tolerability and adverse drug reactions. Respirology, 2017. 22(6): p. 1171-1178.

35. Bando, M., et al., Clinical Experience of the Long-term Use of Pirfenidone for Idiopathic Pulmonary Fibrosis. Internal Medicine, 2016. 55(5): p. 443-448.

36. Ntolios, P., et al., Longitudinal outcomes of patients enrolled in a phase Ib clinical trial of the adiposederived stromal cells-stromal vascular fraction in idiopathic pulmonary fibrosis. Clin Respir J, 2018. 12(6): p. 2084-2089.

37. Felix, R.G., et al., Adipose-derived stem cells and adipose-derived stem cell-conditioned medium modulate in situ imbalance between collagen I- and collagen V-mediated IL-17 immune response recovering bleomycin pulmonary fibrosis. Histol Histopathol, 2020. 35(3): p. 289-301.

38. Reddy, M., et al., Human Adipose-derived Mesenchymal Stem Cells Attenuate Early Stage of Bleomycin Induced Pulmonary Fibrosis: Comparison with Pirfenidone. Int J Stem Cells, 2016. 9(2): p. 192-206.

39. Tashiro, J., et al., Therapeutic benefits of young, but not old, adipose-derived mesenchymal stem cells in a chronic mouse model of bleomycin-induced pulmonary fibrosis. Transl Res, 2015. 166(6): p. 554-67.

40. Gao, Y., et al., Extracellular Vesicles Derived from Adipose Mesenchymal Stem Cells Alleviate PM2.5Induced Lung Injury and Pulmonary Fibrosis. Med Sci Monit, 2020. 26: p. e922782.

41. Chen, S., et al., Transplantation of adipose-derived mesenchymal stem cells attenuates pulmonary fibrosis of silicosis via anti-inflammatory and anti-apoptosis effects in rats. Stem Cell Res Ther, 2018. 9(1): p. 110.

42. Wang, Z., et al., GDNF enhances the anti-inflammatory effect of human adipose-derived mesenchymal stem cell-based therapy in renal interstitial fibrosis. Stem Cell Res, 2019. 41: p. 101605.

43. De Luna-Saldivar, M.M., et al., Advantages of adipose tissue stem cells over CD34(+) mobilization to decrease hepatic fibrosis in Wistar rats. Ann Hepatol, 2019. 18(4): p. 620-626. 
44. Chai, C.Y., et al., Adipose tissue-derived stem cells inhibit hypertrophic scar (HS) fibrosis via p38/MAPK pathway. J Cell Biochem, 2019. 120(3): p. 4057-4064.

45. Song, Y., et al., Adipose-derived stem cells ameliorate renal interstitial fibrosis through inhibition of EMT and inflammatory response via TGF-beta1 signaling pathway. Int Immunopharmacol, 2017. 44: p. 115-122.

46. Fikry, E.M., W.A. Hassan, and A.M. Gad, Bone marrow and adipose mesenchymal stem cells attenuate cardiac fibrosis induced by methotrexate in rats. J Biochem Mol Toxicol, 2017. 31(11).

47. Chen, W., et al., Adipose tissue-derived stem cells ameliorates dermal fibrosis in a mouse model of scleroderma. Asian Pacific Journal of Tropical Medicine, 2017. 10(1): p. 52-56.

48. Sangkum, P., et al., Effect of adipose tissue-derived stem cell injection in a rat model of urethral fibrosis. Can Urol Assoc J, 2016. 10(5-6): p. E175-E180.

49. Ortiz, L.A., et al., Mesenchymal stem cell engraftment in lung is enhanced in response to bleomycin exposure and ameliorates its fibrotic effects. Proceedings of the National Academy of Sciences of the United States of America, 2003. 100(14): p. 8407-8411.

50. Lee, S.H., et al., The effect of adipose stem cell therapy on pulmonary fibrosis induced by repetitive intratracheal bleomycin in mice. Exp Lung Res, 2014. 40(3): p. 117-25.

51. Kolahian, S., et al., Immune Mechanisms in Pulmonary Fibrosis. Am J Respir Cell Mol Biol, 2016. 55(3): p. 309-22.

52. Ganguly, K., et al., Secreted Phosphoprotein 1 Is a Determinant of Lung Function Development in Mice. American Journal of Respiratory Cell and Molecular Biology, 2014. 51(5): p. 637-651.

53. Pardo, A., et al., Up-Regulation and Profibrotic Role of Osteopontin in Human Idiopathic Pulmonary Fibrosis. PLOS Medicine, 2005. 2(9): p. e251.

54. Zhu, Z., et al., TREM2 suppresses the proinflammatory response to facilitate PRRSV infection via PI3K/NF-KB signaling. PLOS Pathogens, 2020. 16(5): p. e1008543.

55. Byers, D.E., et al., Triggering Receptor Expressed on Myeloid Cells-2 Expression Tracks With M2-Like Macrophage Activity and Disease Severity in COPD. Chest, 2018. 153(1): p. 77-86.

56. Turnbull, I.R., et al., Cutting Edge: TREM-2 Attenuates Macrophage Activation. The Journal of Immunology, 2006. 177(6): p. 3520-3524.

\section{Figures}




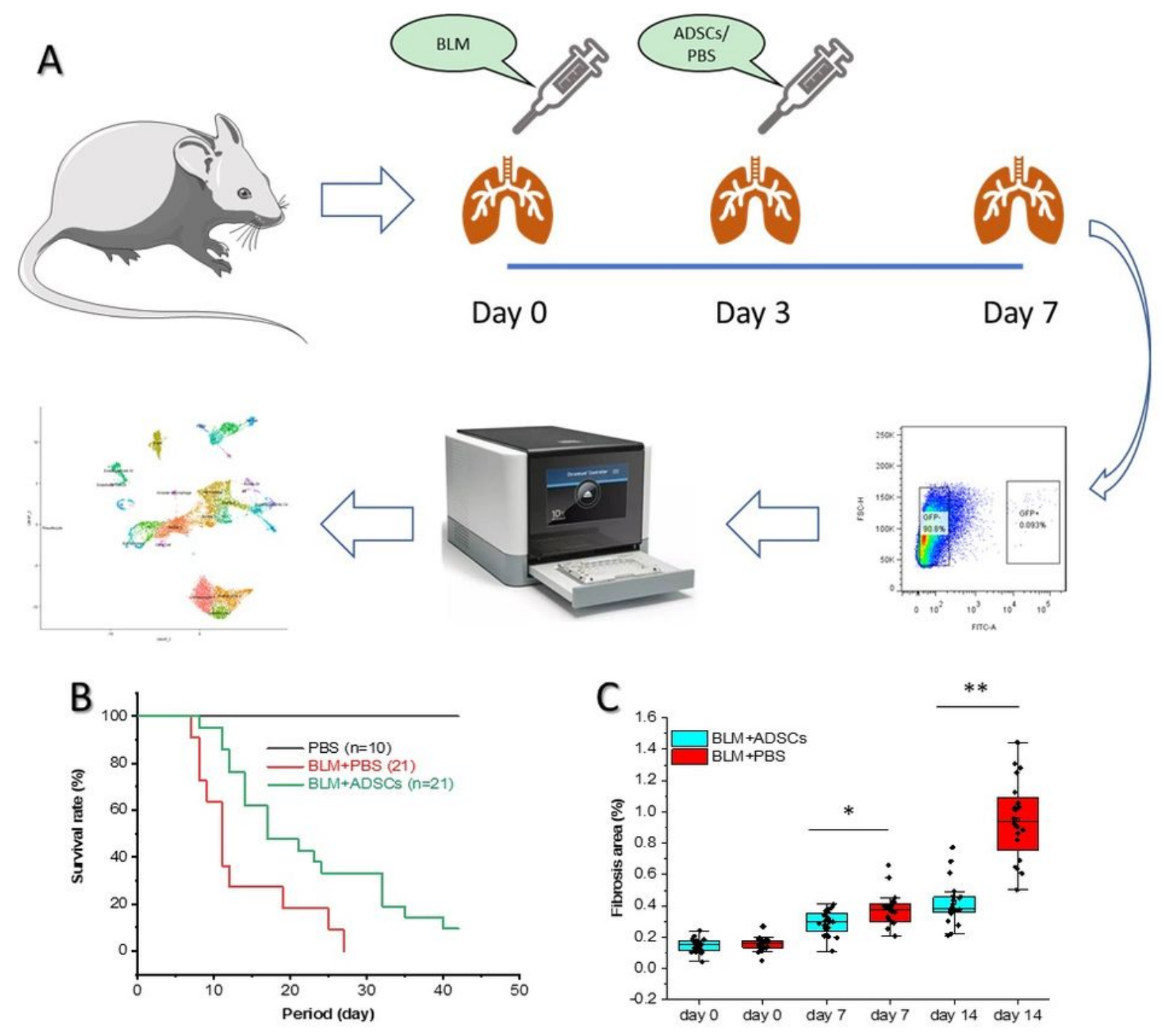

D

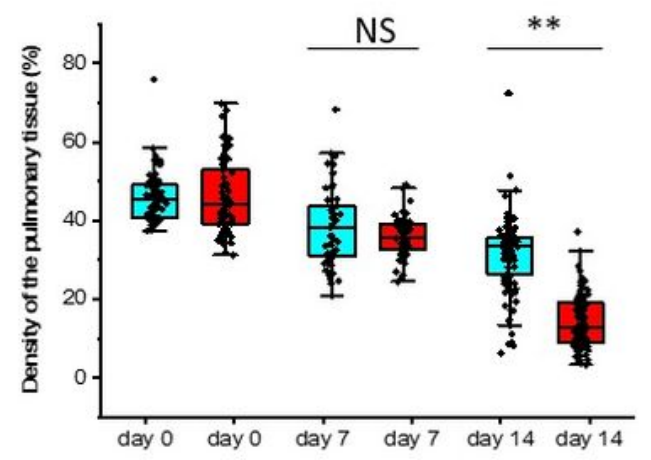

\section{Figure 1}

injection of mADSCs through intratracheal delayed the progression of pulmonary fibrosis. (a) the schematic diagram of BLM lung injury and mADSCs injection through the intratracheal, and tissue/cell collection for single-cell RNA analysis. (b) Kaplan-Meier survival curve for pulmonary fibrosis mice. The group was received PBS ( $n=10$, black line), the BLM+PBS group administered $3.5 \mathrm{mg} / \mathrm{kg} B L M$ in 50 ul PBS at day 0 ( $n=21$, red line), BLM+mADSCs group received $5 \times 10^{\wedge} 5$ cell in 50 ul PBS by intratracheally at 
day 3 post-BLM administration. (c) The degree of pulmonary fibrosis area, on histological sections of Masson's trichrome staining, was measured by IPP software. (d) The density of the pulmonary tissue. on histological sections of H\&E trichrome staining, was measured by IPP software. Data are graphed as mean \pm SD. ${ }^{*} P<0.05 ; * * P<0.01 ; * \star * p<0.001$.
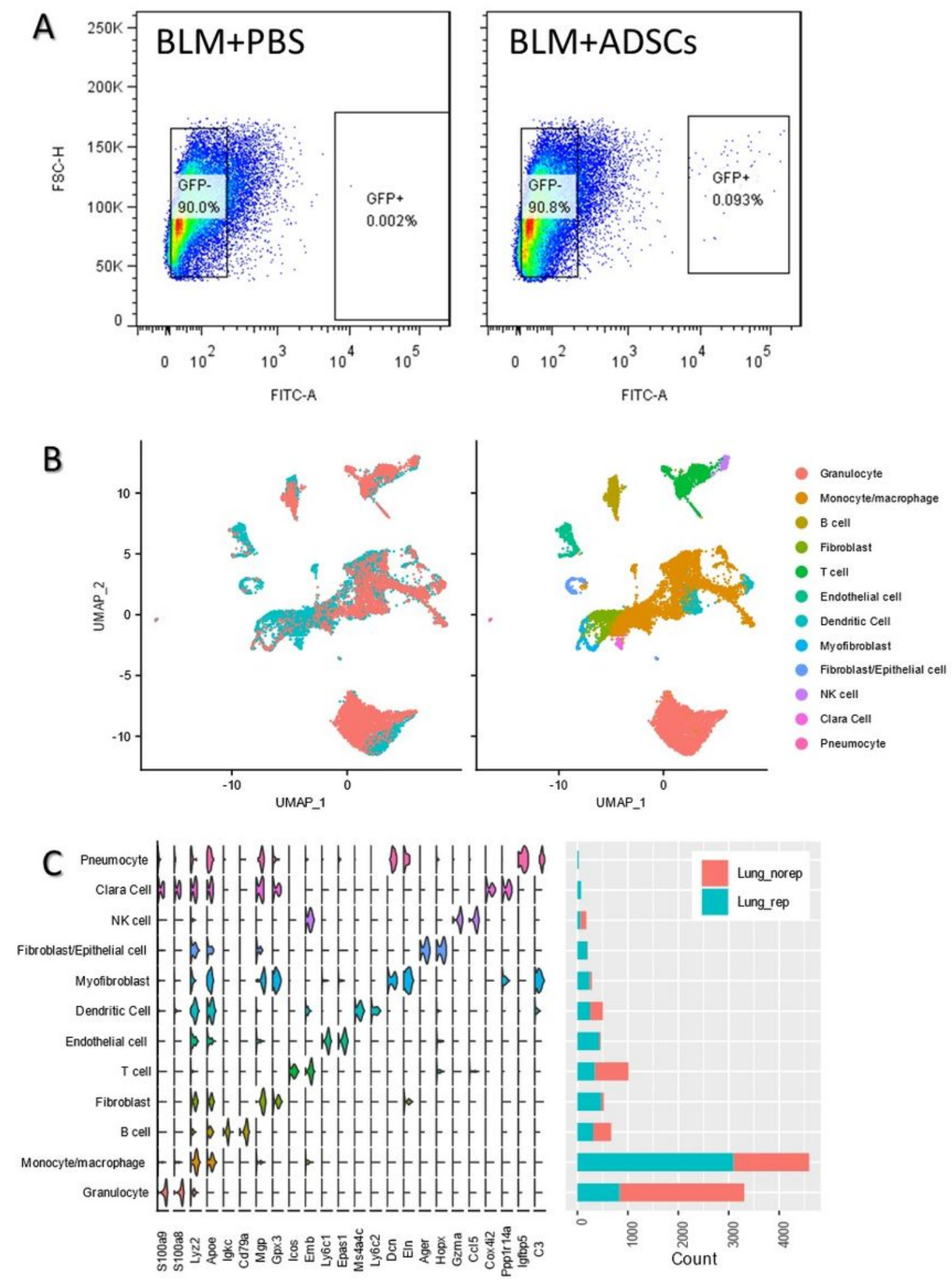

Figure 2 
injection of mADSCs through the intratracheal caused increase of monocyte/macrophage population. (a) The flow cytometry diagrams for cell sorting of lung parenchyma cells (GFP-) and retrieved mADSCs (GFP+), left is BLM+PBS and right is BLM+mADSCs group. (b) Single-cell analysis of lung parenchyma cells, blue is BLM+mADSCs group and red is BLM+PBS group (left UMAP chart), both groups clustered 12 cell types (right UMAP chart). (c) identification of cluster markers (three marker genes for each cluster) and cell count for each cluster of lung parenchyma cells.

A
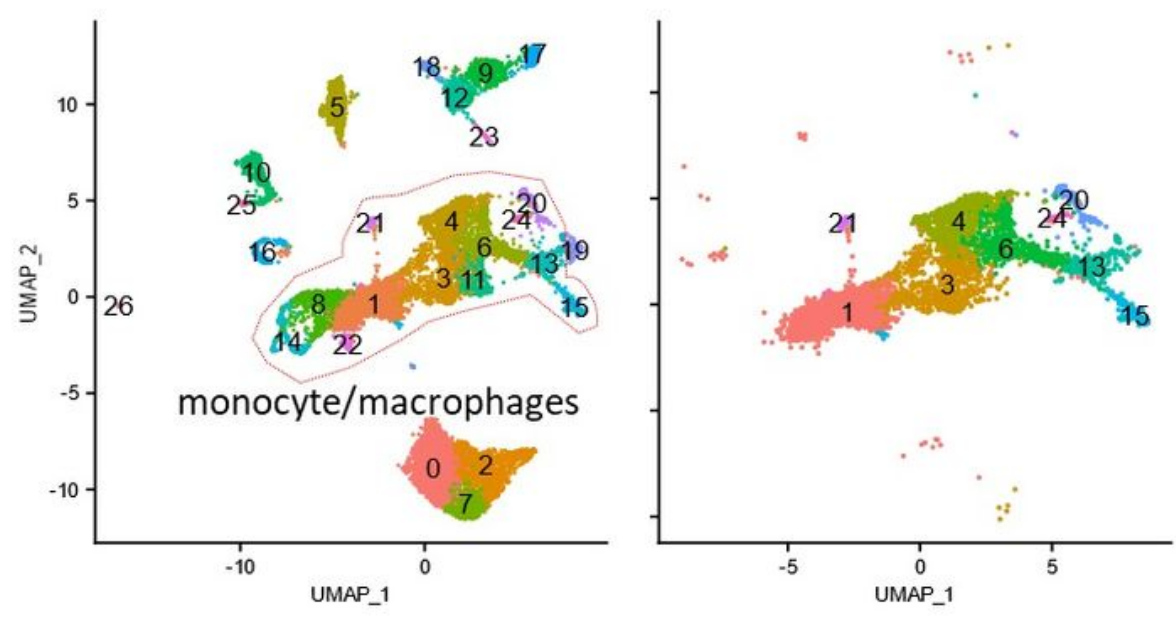

B

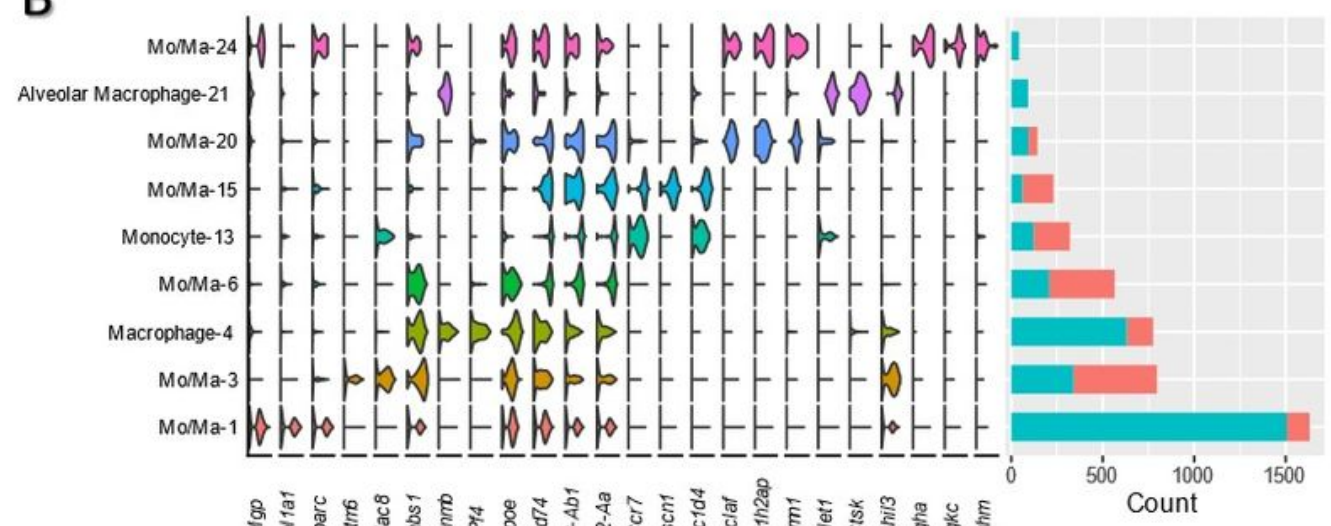

C

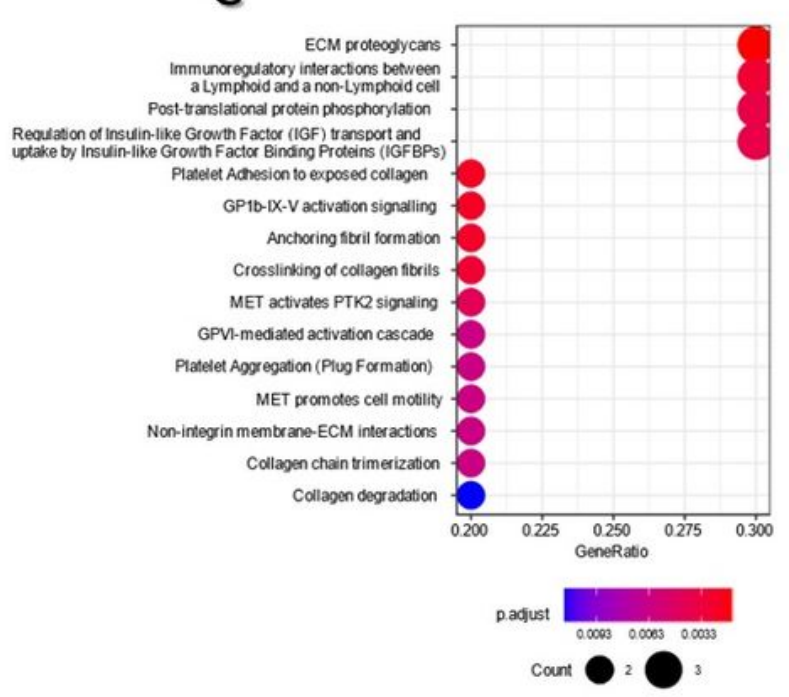

D

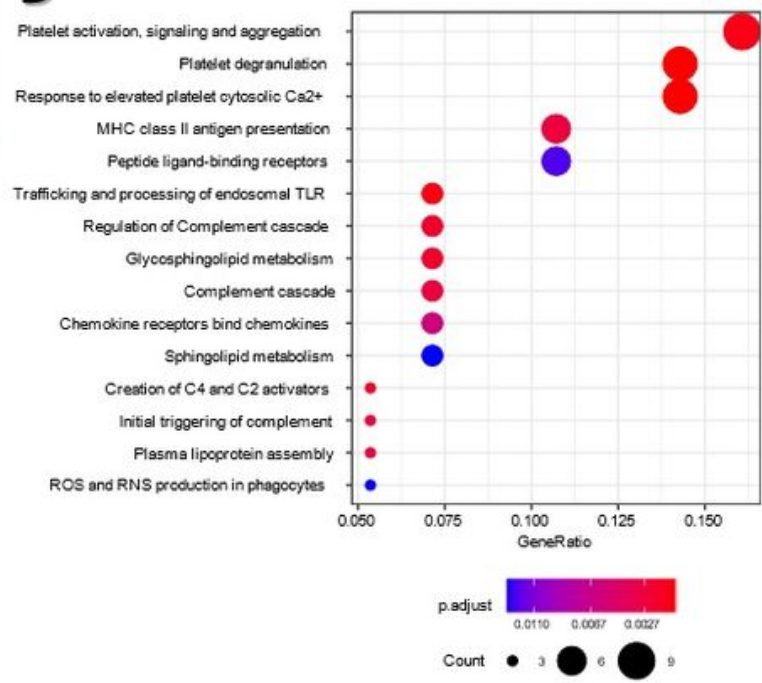

Figure 3 
Go enrichment and KEGG analysis of monocyte/macrophage (a) UMAP distribution of 9 monocyte/macrophage clusters in both groups. (b) identification of sub-cluster markers (three marker genes for each cluster) and cell count of monocyte/macrophage. (c) GO analysis of 9 monocyte/macrophage cells. (d) KEGG analysis of 9 monocyte/macrophage cells.
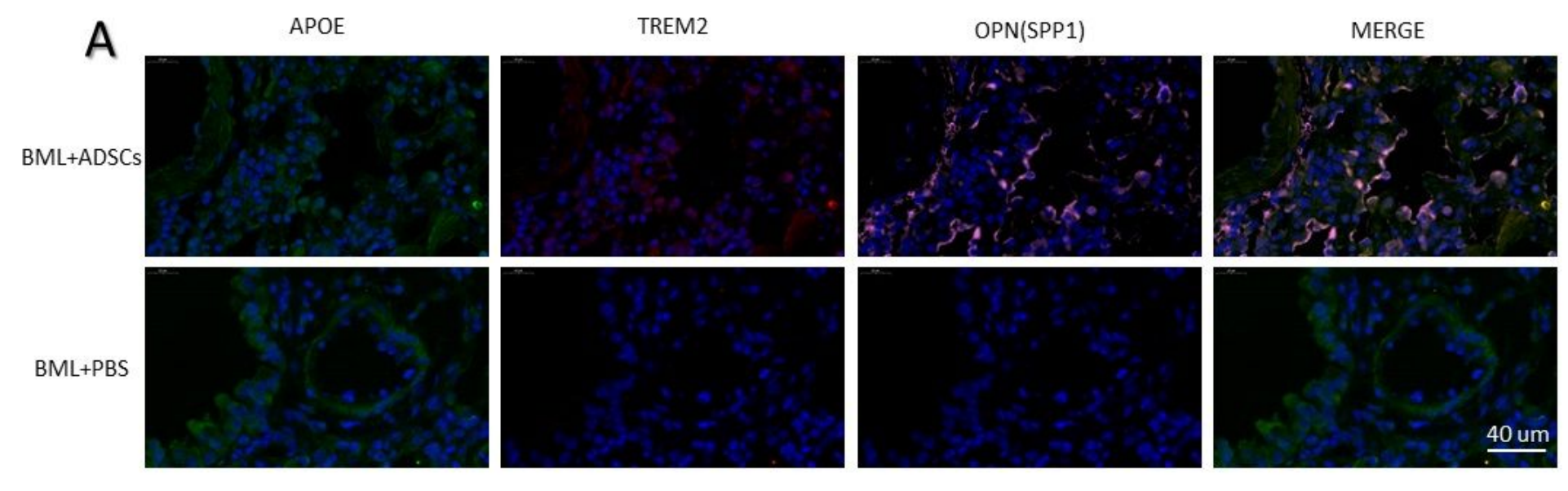

B

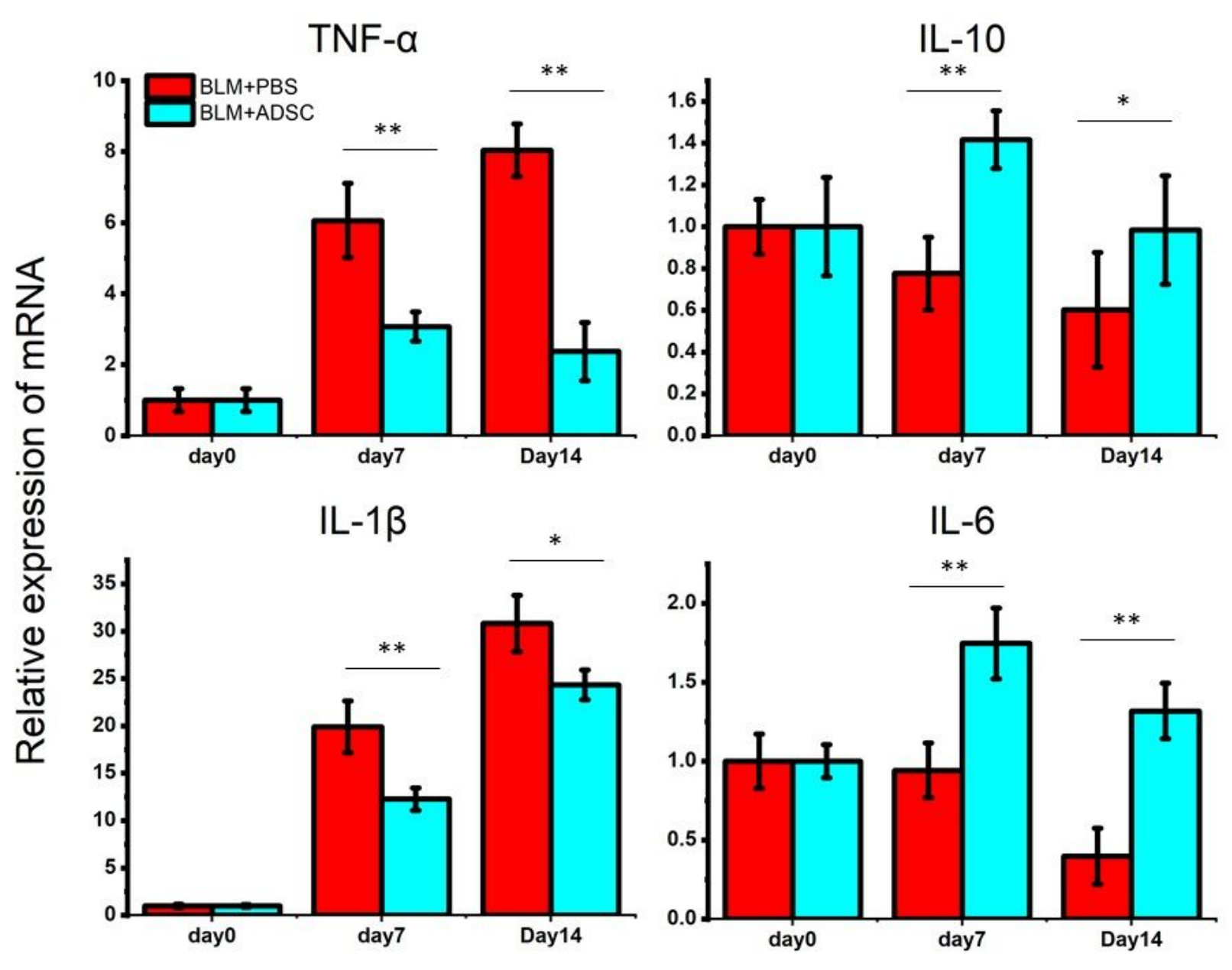

Figure 4

injection of mADSCs through intratracheal caused suppressed of the inflammation in the mice lung tissues (a) The immunofluorescence images of APOE, TREM2, and SPP1 which the high expression 
genes in macrophage-4 on day 7 , on day 0 BLM treatment, and day 3 PBS/mADSCS. (b) The mRNA expression of inflammation and anti-inflammation markers, TNF-a, IL-1 $\beta, \mathrm{IL}-10$, and IL- 6 on day 7 were determined by RT-qPCR. Data are graphed as mean \pm SD. ${ }^{*} P<0.05 ;{ }^{* *} \mathrm{P}<0.01$.

\section{Supplementary Files}

This is a list of supplementary files associated with this preprint. Click to download.

- Suppl1.jpg

- Suppl2.jpg

- Suppl3.jpg 